\title{
Giant uterine leiomyoma in a pregnant woman - case study and review of literature
}

\author{
Kinga KadelskaA, Małgorzata SokołowskaB, Sebastian Kwiatkowskic, Joanna Lebdowicz-KnulD, \\ Wioletta Mikołajek-Bedner ${ }^{\mathrm{E}}$, Yousef Al-HeibF, Andrzej Torbé ${ }^{\mathrm{G}} \bowtie$
}

Pomeranian Medical University in Szczecin, Department of Obstetrics and Gynecology, Powstańców Wlkp. 72, 70-111 Szczecin, Poland

A ORCID: 0000-0002-3613-5113; $\quad{ }^{\text {B } O R C I D: ~ 0000-0001-7707-5498 ; ~} \quad$ C ORCID: 0000-0003-1504-3639; $\quad$ D ORCID: 0000-0002-8447-3539;

E ORCID: 0000-0003-4425-0143; F ORCID: 0000-0003-1726-2691; G ORCID: 0000-0002-6344-4798

\torbea@wp.pl

\begin{abstract}
The case of successful pregnancy outcome in a 39-year-old nulligravida with an enormous leiomyoma is presented. At 16th week of gestation patient began to complain of dyspnoea and abdominal pain. Due to rapid growth, high vascularization, worsening pain, respiratory symptoms and limited conditions for foetal development, surgery was taken. Repeat sonography was performed at the 20th week and normal fetal development consistent with a 2nd trimester pregnancy was observed. Singular fibroids $1-5 \mathrm{~cm}$ in diameter were visualized and no tumor
\end{abstract}

growth was noted when compared with subsequent ultrasound studies. At the 39th week, the patient was referred to the hospital for preparations towards elective cesarean section due to a breech presentation. During the planned cesarean section, numerous subscapular fibroids were observed which were left without intervention. The patient delivered a live female neonate, in a good condition, weighting $3280 \mathrm{~g}$. There were no complications during the surgery and the postpartum period. Keywords: uterine leiomyoma; pregnancy complications.

\section{INTRODUCTION}

Uterine fibromyomas, also known as fibroids or leiomyomas, are the most commonly occurring tumors of the female reproductive tract [1]. They develop in women of childbearing age and can often occur during pregnancy [2]. According to previously published data, the incidence of uterine fibroids diagnosed by USG during pregnancy ranges from $0.1-12.5 \%[1,3]$. These figures are greatly influenced by the age of the mother [4]. In comparison with multiparous women, nulliparous females are 4 times more likely to develop uterine leiomyomas.

Three key factors greatly influence the formation and growth of fibroids: genetic defects (mutations of HMG1, HMG2, fumarate hydratase) [4, 5], steroid sex hormones (estrogen and progesterone), and angiogenic growth factors, such as vascular endothelial growth factor (VEGF) [4, 5, 6, 7]. During pregnancy, increased concentrations of sex hormones may lead to substantial uterine fibroid growth, which could potentially result in worsening symptomatic pain caused by the compression of neighboring structures or fibroid degeneration. Uterine fibromyomas are also a common cause of primary and secondary infertility [8].

Fibroid presence during pregnancy doubles the incidence of pregnancy complications such as spontaneous miscarriage, preterm labor, suboptimal fetal and placental positioning, intrauterine growth restriction, abnormal uterine contractions and perinatal hemorrhage $[2,4,8,9]$.

\section{CASE HISTORY}

A 39-year old nulliparous female in her 16th week of pregnancy was referred to the Department of Obstetrics and Gynecology of Pomeranian Medical University in Szczecin (in Poland) following progressive, substantial tumor growth resulting in a fibroid diameter of approx. $20-22 \mathrm{~cm}$. The patient was overweight (grade $1, \mathrm{BMI}=34.63$ ) and generally in good health. Daily medications included acetylsalicylic acid $(75 \mathrm{mg})$, dydrogesterone $(10 \mathrm{mg}$ ) and prenatal vitamins. The patient reported 3 spontaneous abortions in the past. The menses before pregnancy were regular, painless, and lasted approx. 6 days. Family history was negative for uterine fibroids.

Due to a complicated obstetric history, a pre-conception USG was performed about 6 month before present pregnancy, and it was negative for uterine anatomical anomalies and fibromyomas. During the first weeks of pregnancy, the patient felt well and the laboratory values were within reference ranges. The first-trimester USG performed at 12th week of pregnancy, showed numerous small uterine fibroids, with one bigger myoma $4 \mathrm{~cm}$ of diameter (Fig. 1).

At approx. the 16th week of gestation, the patient began to complain of pain in the umbilical and epigastric area which was characterized by a feeling of heaviness and spreading. A follow-up USG was performed and revealed a tumor, most likely a uterine fibroid, approx. $18 \mathrm{~cm}$ in diameter. The patient did not previously require any pain medication; however, the intensifying pain and tightness resulting from tumor growth were grounds for admission to the ward. 


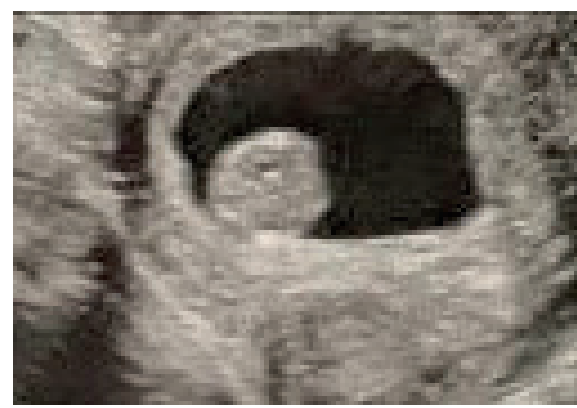

FIGURE 1. One bigger fibroid in the myomatous uterus at 12 week of pregnancy

On admission to the hospital, the patient was in good general condition with normal circulatory and respiratory function. An USG confirmed the presence of a live pregnancy and described a massive tumor, most likely a uterine leiomyoma arising from the fundus, with a diameter of approx. $20 \mathrm{~cm}$. Physical examination revealed a palpable, hard, immovable mass reaching the costal arch, located in the mesogastric and the left hypochondirum areas. The uterus, displaced to the right, together with the tumor filled the entire abdominal cavity causing steadily increased skin tension and elevating the diaphragm. The patient reported worsening dyspnea, platypnea and moderate, occasional abdominal pain. A transvaginal USG showed that the cervical canal was wider than $4 \mathrm{~cm}$ with an external orifice in the shape of the letter T. Laboratory studies indicated normal blood count, morphology, electrolytes, coagulation and urinalysis. C-reactive protein (CRP) levels were elevated to approx. $30 \mathrm{mg} / \mathrm{L}$. Lactate dehydrogenase (LDH) levels increased to 300U/L. Cancer antigen 125 (CA 125) was above normal measuring $191 \mathrm{U} / \mathrm{mL}$ ang human epididymis protein 4 (HE-4) level was $40 \mathrm{pmol} / \mathrm{L}$. Using the risk of ovarian malignancy algorithm (ROMA), the risk of ovarian cancer was concluded to be low.

Due to rapid tumor growth, high vascularization visible on ultrasonography suggesting the diagnosis of a fibroid, as well as worsening pain and respiratory symptoms, a lack of supportive treatment options and limited conditions for further fetal development, surgery treatment was suggested. To remove the uterine fibroid, a laparotomy was proposed. The patient was informed of the benefits and risks of the procedure along with the potential future management options resulting from unfavorable histopathological findings. Methods of extracting the fibroid from the peritoneal cavity, the risk to the pregnancy and uterine loss were also explained. Despite a laden obstetric past, the patient consented to the laparotomy. A midline vertical incision from the pubic symphysis to the xiphoid process was used to separate the abdominal layers and open up the abdominal cavity. Operative findings included a hyperaemic uterus that was shifted to the right side and the size was suitable for 17 weeks of gestation. A few subserosal-intramural fibroids, roughly $5 \mathrm{~cm}$ in diameter, were located within the muscular wall of the uterus (Fig. 2). A solid pedunculated tumor measuring $30 \mathrm{~cm}$ in diameter, reaching to the area of the spleen and stomach, was situated on the left uterine horn. The surface of the tumor was uneven and highly vascularized (Fig. 3).

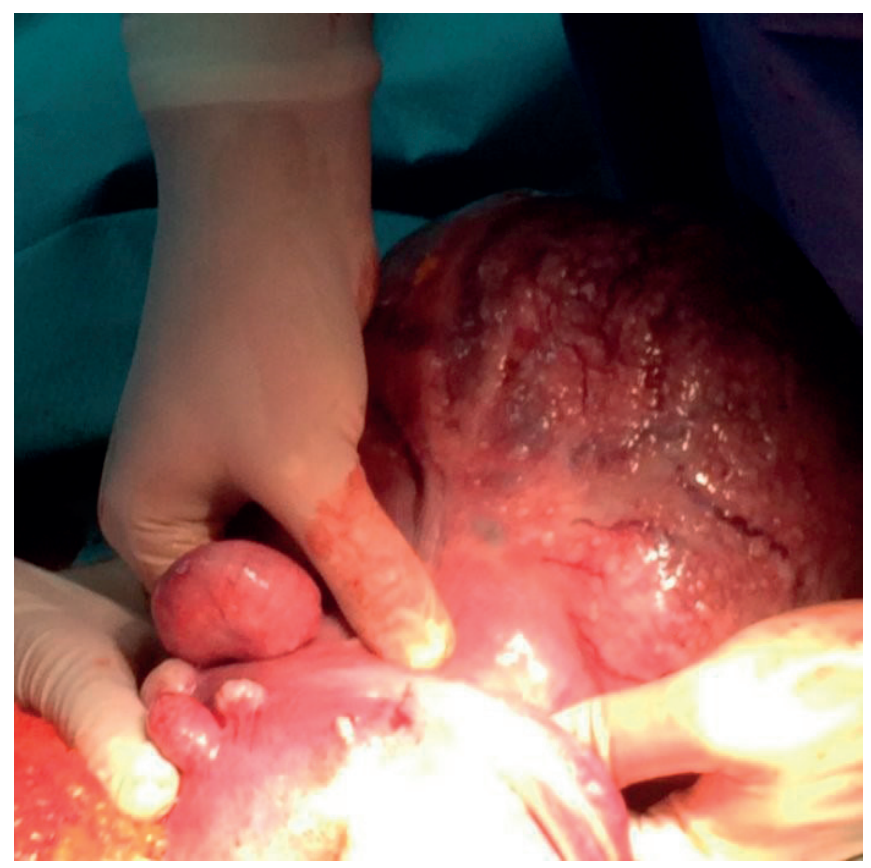

FIGURE 2. Subserosal-intramural fibroids located within the muscular wall of the uterus

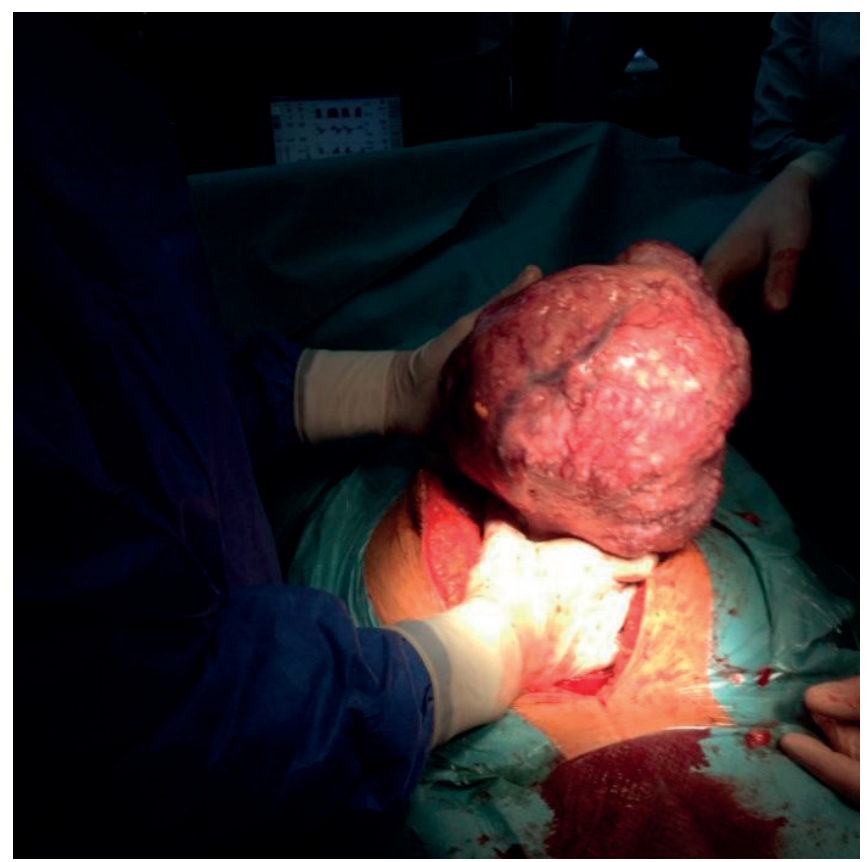

FIGURE 3. Highly vascularized surface of the tumor

The abdominal peritoneum remained smooth, shiny while the uterine appendages remained macroscopically unchanged. The stalk of pedunculated myoma was tied and the tumor was cut from the uterine horn. The uterus was sutured using the Z-suture technique. A fibrin sealant sponge with human fibrinogen and thrombin (TachoSil) was inserted in place of the tumor. Laboratory testing of coagulation was performed and a drain was placed into the Douglas cavity. The abdominal cavity was closed and sutured. There was little blood loss, approx. $200 \mathrm{~mL}$, mainly blood circulating within the tumor. 
Following the surgery, the presence of fetal heart beat was confirmed. The patient was infused with intravenous magnesium sulphate to prevent uterine contractions. Post-operative morphology on the evening of surgery showed a decrease in hemoglobin concentration 12.0-8.0 g/dL and a decrease in hematocrit $34-24 \%$. The patient was in good general condition with a blood pressure of 90/50 mmHG and a heart rate of 90 beats/min. Physical examination revealed a swollen, slightly tender abdomen, negative for peritoneal symptoms. By the drain $200 \mathrm{~mL}$ of bloody fluid was collected. Abdominal ultrasound was positive for $10 \mathrm{~cm}$ of fluid in the area of the liver. The patient was transfused 2 units of red blood cells and 3 units of fresh frozen plasma. Haemostatic therapy was initiated.

Ultrasound examinations were performed at intervals, every several hours, and a stable amount of fluid was described within the peritoneal cavity, and also in the drain. The patient was mobilized on the 1st post-operative day. A decision was made to transfuse 2 additional units of red blood cells. Diuresis remained normal. Laboratory tests showed no significant changes in the coagulative studies, hypoalbuminemia (33 g/L), and anemia (Hgb $6.5 \mathrm{mmol} / \mathrm{L}$, Hct 29\%).

In the 2nd post-operative day, abdominal examination revealed a soft, painless abdomen on palpation, normal peristalsis, and normal uterine tone. Pulse parameters, blood pressure, and temperature were within reference ranges. The drain was removed and a $750 \mathrm{~mL}$ hematoma was evacuated from the site of the drain. A control morphology was performed without significant findings in relation to the preceding results. Due to increased inflammatory markers - CRP $221 \mathrm{mg} / \mathrm{L}$, procalcitonin (PCT) $19 \mathrm{ng} / \mathrm{mL}$ - an infection at the surgical incision site was suspected. Wound swabs were taken and empiric antibiotic therapy, cephalosporin II (cefuroxime), was initiated. Control laboratory studies performed during the following post-operative days revealed a significant decrease of inflammatory markers. No pathological flora was cultured from the wound swab. The incision site was healing properly.

The patient, with a viable pregnancy, was discharged from the hospital in good condition on the 7 th post-operative day. Continued treatment with acetylsalicylic acid, dydrogesterone, enoxaparin, and paracetamol (if needed) was recommended. The patient was referred to the Pathology of Pregnancy and Women's Diseases Clinic for further obstetric care. Histopathological examination of tumor sections were consistent with a leiomyoma with adenomyosis.

Repeat sonography was performed at the 2oth week of gestation and normal fetal development consistent with a 2nd trimester pregnancy was observed. Singular fibroids $1-5 \mathrm{~cm}$ in diameter were also visualized and no tumor growth was noted when compared with subsequent USG. At the 39th week of gestation, the patient was referred to the Department of Obstetrics and Gynecology for preparations towards an elective cesarean section due to a breech fetal presentation. During the planned cesarean section, numerous subscapular fibroids were observed which were left without intervention. The patient delivered a live female neonate weighing $3280 \mathrm{~g}$ with Apgar scores of 9/10/10 respectively. There were no complications during the surgery and the post partum period.

\section{DISCUSSION}

Pregnant women with uterine fibroids are at an increased risk of obstetric complications and require intensive monitoring of both fetal development and fibroid changes. Close monitoring includes regular evaluation of the fibroid for its possible growth, appearance of necrosis or bleeding, its relation to the developing fetus and abdominal organs.

It is not easy to predict the dynamics of fibroid growth in pregnancy as well as the possibility of obstetric complications [10]. Due to possible obstetric complications resulting from the presence of large fibroids or fibrotic uterus in a woman planning a pregnancy, preventive measures such as consideration of any possible surgical intervention in the pre-conception period should be considered. Awareness of the potential risks resulting from the impact of surgical procedures on pregnancy and childbirth should prompt a thorough examination of the patient that is planning to become pregnant as well as hysteroscopic or laparoscopic intervention or abdominal surgery $[11,12]$.

The enlargement of fibroids is noticeable especially in the 1st half of pregnancy [13]. This can be due to a significant decrease in the number of estrogen receptors due to persistence of high estrogen levels and epithelial growth factor (EGF) [14]. In the 2nd and 3rd trimesters of pregnancy, the most common manifestations include the feeling of heaviness, bloating, dull abdominal pain - associated with the presence of enlarged uterine fibroids putting pressure on adjacent abdominal organs. Occasionally degenerative changes in the myoma occur causing severe pain, nausea, vomiting and even fever resulting in urgent hospitalization and intense pain management. The analgesic options in pregnancy are limited [15]. However the management of choice in pregnant women with symptomatic uterine fibromas is conservative treatment as recommended by most referral centers [16]; but sometimes a surgical treatment during pregnancy should be also taken into consideration $[16,17,18,19]$. Surgical removal of the fibroid during pregnancy is associated with a high risk of miscarriage and even uterine damage. High vascularization of the organ places the patient at high risk of massive hemorrhage. Myomectomy during pregnancy could result in damage of the uterine muscle and it increases the risk of uterine rupture during the course of pregnancy and labor. Danzer et al. reported that in about $2 \%$ of pregnancies complicated by uterine leiomyomas, conservative treatment appears to be ineffective and surgical treatment becomes necessary, mainly because of persistent severe pain or bleeding into the peritoneal cavity [20]. However, intraperitoneal bleeding or signs of fetal compression are rare indications for invasive treatment. Kasum analyzed the case of successful delivery of a pregnancy in a 37-year-old patient with a myomectomy at 15 weeks of gestation due to massive intra-peritoneal bleeding from degenerative uterine leiomyomata [21]. The authors 
suggest that fibroid hemorrhage is usually preceded by traumatic or spontaneous inversion of the uterine fibroid. In the described case bleeding occurred after a gynecological examination. Lolis et al. analyzed 622 pregnant women with uterine fibroids of whom 13 required surgery for severe fibroid growth, discomfort and lack of response to painkillers. In this study $92 \%$ of the patients that were treated with surgical intervention carried their pregnancies to term without further complications [17]. Isabu et al. described the case of a 28 -year-old at 14 weeks of gestation complicated by a giant uterine fibroid reaching the right hypochondriac space, where elective caesarean section was performed at 37 weeks of gestation due to placenta previa [22].

Suppurating fibroid is a relatively rare but serious complication in pregnancy. Kobayashi et al. presented the case of a 28-year-old nulliparous woman who was treated with laparotomy during the 21st week of gestation due to a suppurating fibroid and peritonitis. The patient successfully carried the pregnancy to term [23]. Wong et al. described a case of spontaneous bleeding into the peritoneal cavity from a $12 \mathrm{~cm}$ subcutaneous uterine fibroid at 8 weeks of gestation. Instead of a myomectomy, haemostatic sutures were used to suture the bleeding vessels. Following the procedure, the patient had no further complications, carried the pregnancy to term and delivered the baby via a natural delivery [24].

Modern radiological diagnostics (USG, Magnetic Resonance Imaging), which help in accurate localization and evaluation of fibroid size and number, as well as advanced surgical techniques allow, under certain circumstances, the consideration of surgical intervention, myomectomy, during pregnancy $[25,26]$. Invasive procedures seem to be advisable for very large, bleeding, suppurating fibroids, which may affect the development of pregnancy or lead to a maternal health complications. Each case should be reviewed individually, the decision for surgical intervention should be made together with the patient, and these operations should only be carried out by a suitably trained team of specialists in the referred health centers.

\section{REFERENCES}

1. Cooper NP, Okolo S. Fibroids in pregnancy - common but poorly understood. Obstet Gynecol Surv 2005;60(2):132-8.

2. Practice Committee of American Society for Reproductive Medicine in collaboration with Society of Reproductive Surgeons. Myomas and reproductive function. Fertil Steril 2008;90(5 Suppl):S125-30.

3. Cook H, Ezzati M, Segars JH, McCarthy K. The impact of uterine leiomyomas on reproductive outcomes. Minerva Gynecol 2010;62(3):225-36.

4. Verkauf BS. Myomectomy for fertile enhancement and preservation. Fertil Steril 1992;58(1):1-15
5. Alam NA, Olpin S, Leigh IM. Fumarate hydratase mutations and predispositions to cutaneous leiomyomas, uterine leiomyomas and renal cancer. Br J Dermatol 2005;153(1):11-7.

6. Parazzini F, La Vecchia C, Negri E, Cecchetti G, Fedele L. Epidemiologic characteristic of women with uterine fibroids: a case-control study. Obstet Gynecol 1988;72(6):853-7.

7. Ciarmela P, Islam MS, Reis FM, Gray PC, Bloise E, Petraglia F, et al. Growth factors and myometrium: biological effects in uterine fibroid and possible clinical implications. Hum Reprod Update 2011;17(6):772-90.

8. Pritts EA, Parker WH, Olive DL. Fibroids and infertility: an updated systematic review of the evidence. Fertil Steril 2009;91(4):1215-23.

9. Bulletti C, de Ziegler D, Levi Setti P, Cicinelli E, Polli V, Stefanetti M. Myomas, pregnancy outcome, and in vitro fertilization. Ann NY Acad Sci 2004;1034:84-92.

10. Bengalia L, Cardellicchio L, Filippi F, Paffoni A, Vercellini P, Somigliana E, et al. The rapid growth of fibroids during early pregnancy. PLoS One 2014;9(1):e85933.

11. Seracchioli R, Manuzzi L, Vianello F, Gualerzi B, Savelli L, Paradisi R, et al. Obstetric and delivery outcome of pregnancies achieved after laparoscopic myomectomy. Fertil Steril 2006;86(1):159-65.

12. Sinclair D, Gaither K, Mason TC. Fertility outcomes following myomectomy in an urban hospital setting. J Natl Med Assoc 2005;97(10):1346-8.

13. De Vivo A, Mancuso A, Giacobbe A, Savasta LM, De Dominici R, Dugo N, et al. Uterine myomas during pregnancy: a longitudinal sonographic study. Ultrasound Obstet Gynecol 2011;37(3):361-5.

14. Bińkowska M, Dębska M, Dębski R. Olbrzymi mięśniak macicy u ciężarnej - opis przypadku. Ginekol Pol 2009;80(12):946-8.

15. Klatsky PC, Tran ND, Caughey AB, Fujimoto VY. Fibroids and reproductive outcomes: a systematic literature review from conception to delivery. Am J Obstet Gynecol 2008;198(4):357-66.

16. Lozza V, Pieralli A, Corioni S, Longinotti M, Penna C. Multiple laparotomic myomectomy during pregnancy: a case report. Arch Gynecol Obstet 2011;284(3):613-6.

17. Lolis DE, Kalantaridou S, Makrydimas G, Sotiriadis A, Navrozoglou I, Zikopoulos K, et al. Successful myomectomy during pregnancy. Hum Reprod 2003;18(8):1699-702.

18. Domenici L, Di Donato V, Gasparri ML, Lecce F, Caccetta J, Panici PB. Laparotomic myomectomy in the 16 th week of pregnancy: a case report. Case Rep Obstet Gynecol 2014;2014:154347.

19. De Carolis S, Fatigante G, Ferrazzani S, Trivellini C, De Santis L, Mancuso $S$, et al. Uterine myomectomy in pregnant women. Fetal Diagn Ther 2001;16(2):116-9.

20. Danzer E, Holzgreve W, Batukan C, Miny P, Tercanli S, Hoesli I. Myomectomy during the first trimester associated with fetal limb anomalies and hydrocephalus in a twin pregnancy. Prenat Diagn 2011;21(10):848-51.

21. Kasum M. Hemoperitoneum caused by a bleeding myoma in pregnancy. Acta Clin Croat 2010;49(2):197-200.

22. Isabu P, Eigbefoh J, Okogbo F, Okunsanya S, Eifediyi R. Myomectomy during second trimester pregnancy: a case report. Niger Postgrad Med J 2010;17(4):324-6.

23. Kobayashi F, Kondoh E, Hamanishi J, Kawamura Y, Tatsumi K, Konishi I. Pyomayoma during pregnancy: a case report and review of the literature. J Obstet Gynaecol Res 2013;39(1):383-9.

24. Wong L, Ching TW, Kok TL, Koon TH. Spontaneous hemoperitoneum from an uterine leiomyoma in pregnancy. Acta Obstet Gynecol Scand 2005;84(12):1208-9.

25. Exacoustòs C, Rosati P. Ultrasound diagnosis of uterine myoma and complications in pregnancy. Obstet Gynecol 1993;82(1):97-101.

26. Strobelt N, Ghidini A, Cavallone M, Pensabene I, Ceruti P, Vergani P. Natural history of uterine leiomyomas in pregnancy. J Ultrasound Med 1994;13(5):399-401. 